\title{
COMPARAÇÃO ENTRE TÉCNICAS DE IMUNOISTOQUÍMICA E DE IMUNOFLUORESCÊNCIA PARA ANTIGENEMIA PP65 DO CITOMEGALOVÍRUS (CMV) EM RECEPTORES DE TRANSPLANTE
}

\author{
Comparison between Immunocytochemical and Immunofluorescence techniques for Cytomegalovirus \\ Antigenemia Detection in Transplant Recipients
}

Regina Barbosa Schroeder ${ }^{1,2}$, Tatiana Michelon ${ }^{1,2}$, João Wurdig², Elizete Keitel', Jorge Neumann²

\begin{abstract}
RESUMO
Infecção por citomegalovírus é importante causa de morbidade em pacientes imunossuprimidos, como os receptores de transplantes. A detecção do antígeno viral através da antigenemia pp65 em sangue periférico tem-se mostrado eficiente como marcador de reativação viral, com boa correlação clínica. Houve algumas modificações na técnica artesanal ao desenvolvimento de kits comerciais, o que padronizou e diminuiu o tempo de execução. Objetivo: Comparar a eficácia diagnóstica de dois kits comerciais que utilizam anticorpo monoclonal primário $\mathrm{C} 10 / \mathrm{C} 11$ específico contra a proteína pp65 da matriz viral do citomegalovírus em amostras de sangue de pacientes transplantados. Material e Métodos: Foram estudadas 132 amostras aleatórias de sangue testadas simultaneamente através dos dois kits comerciais para pesquisa de antigenemia pp65 do CMV: a) Imunoistoquímica (IQ), que usa uma anti-fosfatase alcalina como revelador -[APAAP-BIOTESTAlemanha] em granulócitos previamente separados com dextran, necessitando de seis horas para execução e, b) Imunofluorescência(IF), que usa fluoresceína [BRITE TURBO-IQP-Holanda] em leucócitos totais, e é realizada em duas horas. Resultado: Os pacientes tinham em média 41,1 $\pm 20,1$ anos e eram receptores de transplantes: renal $(\mathrm{n}=73 ; 55,3 \%)$, pulmonar $(\mathrm{n}=34 ; 25,8 \%)$, hepático $(\mathrm{n}=11 ; 8,3 \%)$, medula óssea $(n=7 ; 5,3 \%)$, rim conjugado ao pâncreas $(n=3 ; 2,3 \%)$ ou cardíaco $(n=4 ; 3,0 \%)$. A mediana de tempo pós-transplante na colheita da amostra para antigenemia foi de 91 dias (5 a 2.103 dias). O emprego da técnica de IQ resultou 35,6\% (n=47) de amostras positivas, sendo essa taxa 38,6\% (n=51) quando avaliada através de IF. A mediana do número de células+ foi de 4,0/100.000 granulócitos na IQ (máximo=515 granulóccitos+) e 5,0/200.000 leucócitos na IF (máximo=682 leucócitos+). Houve discordância em 4,5\% (6/130) entre eles, cinco foram testes falso-negativos pela IQ, que foram considerados positivos pela IF (mediana=1cél+/200.000 leucócitos); o outro caso foi um falsonegativo de IF, com resultado positivo pela IQ (1cél+/100.000 granulócitos). A correlação foi altamente significativa ( $\mathrm{P}=0,000)$ entre as duas técnicas, tanto entre testes positivos $(\mathrm{R}=0,974)$ como na análise global $(\mathrm{R}=0,996)$. Conclusão: Antigenemia por Imunoistoquímica e Imunofluorescência apresentam correlação altamente significativa para pesquisa de antigenemia pp65 para CMV pós-transplante, sendo a IF mais rápida e de mais fácil execução.
\end{abstract}

Descritores: Transplante de órgãos, Infecções por Citomegalovírus, Citomegalovírus, Diagnóstico, Imunofluorescência, Imunoistoquímica.

\footnotetext{
Instituições:

1 Programa de Pós-Graduação em Patologia - Universidade Federal de Ciências da Saúde de Porto Alegre

${ }^{2}$ Laboratório de Imunologia de Transplantes - Santa Casa de Porto Alegre
}

Correspondência:

Regina Barbosa Schroeder

Hospital Dom Vicente Scherer

Av. Independência 75 - Porto Alegre / RS - CEP: 94035-074 - Brasil

Tel/Fax: (51) $32148670 / 32148629$

E-mail: regina@santacasa.tche.br

\section{INTRODUÇÃo}

O Citomegalovírus (CMV) é um beta-herpes vírus que causa infecção primária em 50 a 100\% da população durante a infância. A primo-infecção geralmente é assintomática, seguida pela excreção do vírus em saliva e outras secreções e permanece em estado de latência principalmente em leucócitos do hospedeiro. A infecção primária ou a reativação da infecção latente pelo CMV são responsáveis por morbimortalidade em pacientes imunossuprimidos, como os receptores de transplante ou portadores da síndrome da imunodeficiência adquirida (SIDA), também ocorrendo após quimioterapia e na gestação. ${ }^{1-4}$

As formas clínicas dependem do tipo e da intensidade da imunossupressão e do tipo de infecção, primária ou secundária. Em pacientes transplantados pode mimetizar sintomas de 
rejeição ao enxerto, especialmente nos primeiros três meses póstransplante, sendo mandatório o diagnóstico diferencial de forma específica e precoce..$^{2,5}$

Apesar de os métodos baseados em amplificação de ácido nucléico terem revolucionado a aplicabilidade clínica do laboratório de virologia na última década, a pesquisa da antigenemia pp65 do CMV vem sendo utilizada em transplantes de órgãos e tecidos desde o final da década de $800^{3,6,7,8}$ Essa metodologia é amplamente utilizada por centros de transplantes do mundo todo ainda nos dias atuais, com considerável sucesso em estratégias de terapia preemptiva, por permitir o diagnóstico de infecção ativa e/ou replicação viral incipiente, evitando com isso o desenvolvimento de doença no órgão-alvo. O teste se baseia na utilização de um anticorpo monoclonal primário contra a proteína pp65 da matriz viral e informa indiretamente a carga viral em células do sangue. ,6-14 $^{3,4}$

O Rio Grande do Sul foi um dos pioneiros no Brasil a utilizar essa técnica para diagnóstico de infecções por CMV para monitorização de receptores de transplante renal. Empregando o método originalmente descrito por van der Bij em 1988 e consolidado a partir de 1993, o Laboratório de Imunologia de Transplantes da Santa Casa de Porto Alegre passou, já naquela época, a reproduzir a sensibilidade e a especificidade observadas na literatura internacional. ${ }^{3,6,15-17}$

O objetivo deste estudo foi comparar a eficácia diagnóstica de dois kits comerciais com métodos distintos de revelação, utilizando o mesmo anticorpo monoclonal primário específico contra a proteína pp65 da matriz viral do CMV (C10/C11), buscando identificar potenciais vantagens técnicas na aplicação rotineira desse teste nos laboratórios clínicos que atendem os serviços de transplante.

\section{PACIENTES E MÉTODOS}

A identificação de antígenos de replicação viral por antigenemia em sangue periférico consiste de três etapas: a) isolamento celular de leucócitos de sangue periférico, b) imunocoloração em lâminas de microscopia, utilizando um anticorpo monoclonal primário e um anticorpo secundário ligado a um revelador (peroxidase, fosfatase alcalina ou fluoresceína) c) leitura através de microscopia óptica (imunoistoquímica) ou de fluorescência (imunofluorescência). ${ }^{7-14}$

Foram analisadas 132 amostras de sangue periférico colhidas com EDTA de pacientes em avaliação de rotina no Laboratório de Imunologia de Transplantes da Santa Casa de Porto Alegre, alocadas entre setembro e outubro de 2005. As amostras foram processadas concomitantemente por dois kits comerciais, de acordo com a recomendação dos fabricantes: a) imunoistoquímica [APAAP-Biotest-Alemanha] e b) imunofluorescência [CMV BRITE TURBO - IQP-Holanda].

A detecção do antígeno pp65 por imunoistoquímica através da revelação pela fosfatase alcalina (APAAP) necessita seis horas para sua execução e utiliza a sedimentação por dextran para separação de leucócitos polimorfonucleares e hemácias a partir de $3 \mathrm{ml}$ de sangue (amostra inicial). O emprego da fluoresceína visualizada através de microscópio de imunofluorescência utiliza a lise de hemácias a partir de uma amostra de $1 \mathrm{ml}$ de sangue, obtendo os leucócitos totais para a análise final, com resultado em até duas horas. O resultado final é expresso em número de células positivas (com replicação viral ativa) em 100.000 granulócitos na técnica de imunoistoquímica e em 200.000 leucócitos na imunofluorescência. ${ }^{3,6,10-15}$

\section{Análise Estatística}

As variáveis foram descritas como média e desvio-padrão ou mediana. A associação entre as variáveis foi analisada através do teste de Quiquadrado ou exato de Fisher, sendo avaliado o coeficiente de correlação de Pearson (IC95\% e P<0,05). O software utilizado para os referidos testes foi Statistical Package for Social Sciences (SPSS versão 14.0).

\section{RESULTADOS}

Os pacientes estudados tinham em média 41,1士20,1 anos e eram receptores de transplantes: renal $(n=73 ; 55,3 \%)$, pulmonar $(n=34$; $25,8 \%)$, hepático $(n=11 ; 8,3 \%)$, célula tronco hematopoiética $(n=7$; $5,3 \%)$, rim conjugado a pâncreas $(n=3 ; 2,3 \%)$ ou cardíaco $(n=4$; $3,0 \%$ ). A mediana de tempo pós-transplante na colheita da amostra para a antigenemia foi de 91dias (cinco a 2103 dias).

Na série analisada, o emprego da técnica de imunoistoquímica resultou em 35,6\% ( $n=47)$ de amostras positivas, sendo essa taxa de 38,6\% $(n=51)$ quando avaliada através de imunofluorescência. A mediana do número de células positivas foi de 4,0/100000 granulócitos na imunoistoquímica (máximo=515 granulócitos positivos) e 5,0/200000 leucócitos na Imunofluorescência (máximo: 682 leucócitos positivos).

Na série analisada, a discordância entre as metodologias ocorreu em seis amostras entre as 132 (4,5\%) amostras analisadas. Entre elas, cinco eram testes falso-negativos por imunoistoquímica, que foram considerados positivos pela imunofluorescência (mediana: 1 cel. positiva em 200000 leucócitos); o outro caso foi um falso-negativo de imunofluorescência, com resultado positivo por imunoistoquímica (1 célula positiva em 100000 granulócitos). A correlação foi altamente significativa $(\mathrm{P}=0,000)$ entre as duas técnicas, tanto entre os testes positivos $(R=0,974)$ como na análise global $(R=0,996)$. A Figura 1 apresenta os resultados acima descritos.

Figura 1. Gráfico de dispersão dos resultados dos testes de antigenemia pp65 para o CMV através das duas metodologias estudadas.

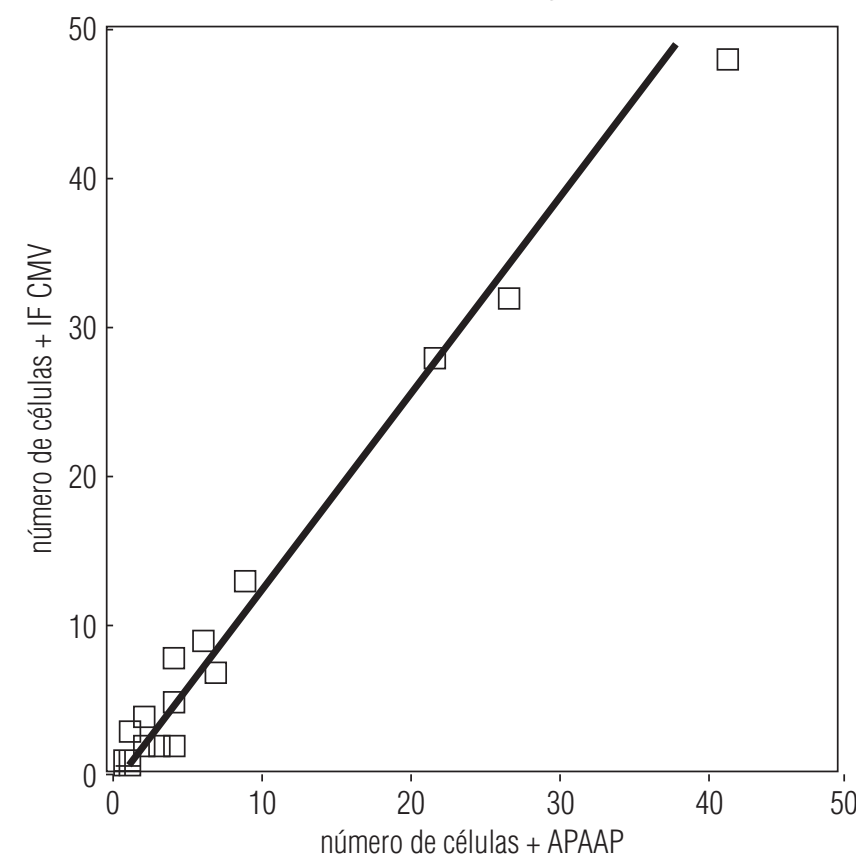

$P=0,000 ; R=0,996$

IF CMV: imunofluorescência para o antígeno pp65 do citomegalovírus APAAP: imunoistoquímica para o antígeno pp65 do citomegalovírus 


\section{DISCUSSÃO}

Diante da alta prevalência de doença por CMV após transplante, da morbidade associada além da toxicidade e do custo dos tratamentos antivirais específicos, é mandatória a monitorização sistemática dessa infecção viral após transplante..$^{4,5,18-21}$ A antigenemia pp65 é uma técnica quantitativa de alta sensibilidade, capaz de disponibilizar resultados em tempo relativamente rápido e que tem apresentado boa reprodutibilidade, especialmente a partir da padronização de reagentes em kits comerciais. Por apresentar tais características, esse teste tem sido amplamente empregado na monitorização de pacientes de risco em estratégias preemptivas e no acompanhamento de terapia antiviral em todo o mundo. ${ }^{5-12}$ No Brasil, estudos em transplante de rim, simultâneo de rim e pâncreas, pulmão, coração, fígado e de células tronco hematopoiética, corroboram sua sensibilidade, especificidade e valor preditivo positivo e negativo para o desenvolvimento de doença em órgãoalvo por CMV após transplante. ${ }^{3,6,16-19}$

Ao longo dos anos, algumas modificações da técnica da antigenemia foram sendo implementadas em busca principalmente de maior simplicidade, rapidez e padronização, para sua utilização mais ampla em laboratórios clínicos. Uma modificação importante foi a introdução da revelação da reação positiva com a fosfatase alcalina e, posteriormente, com fluoresceína. Anteriormente, a revelação era por peroxidase, necessitando a preparação de vários reativos considerados instáveis no momento do processamento da amostra e levando cerca de 24 horas para sua realização. ${ }^{3,6,16-19}$

Modernamente, outras metodologias quantitativas, como PCR em tempo real vêm se tornando cada vez mais disponíveis para os mesmos propósitos de diagnóstico precoce de replicação viral. Cargas virais acima de 5000 cópias/ml de sangue total têm sido valorizadas e sugeridas como indicação de terapia antiviral em alguns importantes centros de transplantes de órgãos sólidos. ${ }^{20-23}$ Todavia, ainda existe necessidade de padronizar reagentes e a técnica propriamente dita, além de extensa correlação clínica da carga viral aos moldes do que se desenvolveu nas últimas décadas

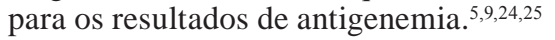

A escolha da melhor metodologia a ser adotada em cada centro deve contemplar, além das características técnicas propriamente ditas (sensibilidade, especificidade e reprodutibilidade) a logística, infra-estrutura disponível e expertise dos profissionais envolvidos. O custo, outro importante determinante dessa escolha é igualmente dependente de diversos fatores, além dos insumos aplicados para a realização do teste e deve ser considerado na melhor relação custo e risco-benefício, nesse caso medido como morbidade e mortalidade após a realização do transplante. A utilização de metodologias diferentes, todavia, pode gerar variações nos resultados obtidos, podendo assumir relevância clínica. Pequenas diferenças, como a observada em favor da imunofluorescência neste estudo, podem ser clinicamente importantes, especialmente em transplantes de células tronco hematopoiética, onde apenas uma célula positiva orienta o manejo clínico para introdução da terapia antiviral preemptivamente. Nesses receptores, quando não se dispõe de acompanhamento laboratorial para identificação de viremia precoce, a mortalidade por doença citomegálica é da ordem de $50 \%$. ${ }^{4,10,25,26,27}$

O presente estudo restringiu-se à comparação de diferentes metodologias empregadas para determinação da antigenemia pp65, justificado pelo período de alocação dos pacientes em nossa instituição. A discreta superioridade da imunofluorescência aqui observada pode ser compensada por maior freqüência da monitorização em pacientes de maior risco em que a cinética da infecção viral é mais rápida. Todavia, apesar de suas vantagens adicionais quando comparada à imunoistoquímica, que incluem maior rapidez, facilidade de execução e necessidade de menor volume de amostra, ambas podem ser igualmente empregadas para o diagnóstico de infecção e de doença citomegálica, bem como para monitorização da terapia antiviral, sem prejuízo clínico. ${ }^{9-15}$

\section{CONCLUSÃO}

Antigenemia por Imunoistoquímica e Imunofluorescência apresentam correlação altamente significativa para a pesquisa de antigenemia pp65 para o CMV em receptores de transplantes.

\section{ABSTRACT}

Introduction: Cytomegalovirus (CMV) infection is a major cause for morbidity among immunossupressed patients in transplant recipients. CMV antigen detection in blood cells by antigenemia test is a good marker for viral reactivation, showing a high clinical correlation. Some improvements has occurred in the last years changing it from an in-house technique to a more practical and standardized test, by the use of commercial kits. Purpose: To make a comparison between immunocytochemical and immunofluorescence techniques to the CMV antigenemia detection in transplant recipients. Methods: It was studied 132 random blood samples from transplant patients, being routinely analyzed for CMV infection. They were simultaneously tested by two different commercial kits for pp65 antigenemia: a) Immunocytochemical (IHC), based on the antigen detection by an anti-alcaline-phosphatase reaction [APAAP-BIOTEST-Germany], and b) immunofluorescence (IF), detecting fluorescein positive reaction [BRITE TURBO-IQP-Netherland]. IHC was performed in previously isolated granulocytes by dextran gradient in a 6-hour turn, and IF was performed in total leukocytes spending about 2 hours. Results: Patients had $41.1 \pm 20.1$ yeas old, and they were recipients of the following transplants: kidney ( $\mathrm{n}=73 ; 55.3 \%)$, lung $(\mathrm{n}=34 ; 25.8 \%)$, liver $(\mathrm{n}=11$; $8.3 \%)$, hematopoietic stem cell $(\mathrm{n}=7 ; 5.3 \%)$, kidney-pancreas $(\mathrm{n}=3 ; 2.3 \%)$ or heart $(\mathrm{n}=4 ; 3.0 \%)$. The median post-transplant follow-up at the time of the sample collection was 91 days (5 to 2,103 days). IHC technique showed $35.6 \%$ ( $\mathrm{n}=47$ ) of positive samples for CMV antigenemia. This rate was $38.6 \%(n=51)$ using IF. The median positive-cells was 4.0/100000 granulocytes by IHC (highest: 515 positive-granulocytes), and 5.0/200000 leukocytes by IF (highest: 682 positive-leukocytes). Disagreement occurred in 6 samples (4.5\%): 5 IQ false-negative were positive by IF (median=1+cell/200000 leukocytes); and 1 false-negative case by IF with positive result when analyzed by IHC (1+cell/100000 granulocytes). The correlation was highly significant $(\mathrm{P}=0.000)$ between both techniques, whether among the positive tests $(\mathrm{R}=0.974)$ or in the general analysis $(\mathrm{R}=0.996)$. Conclusion: Immunocytochemical or immunofluoresce antigenemia is suitable to the pp65 $\mathrm{CMV}$ antigen detection in transplant recipients. Regarding the high correlation between them, IF has the advantage to be easier and faster.

Keywords: Organ Transplantation, Cytomegalovirus Infections, Cytomegalovirus, Diagnosis, Fluorescent Antibody Technique, Immunohistochemistry. 
1. Drago F, Aragone MG, Lugani C, Rebora A. Cytomegalovirus infection in normal and immunocompromised humans. A review. Dermatology. 2000;200:189-95.

2. De Jong MD, Galasso GJ, Gazzard B, Grif PD, Jabs DA, Kern ER, et al. Summary of the II International Symposium on Cytomegalovirus. Antiviral Res. 1998; 39:141-62.

3. Deboni L. Estudo da incidência da infecção por citomegalovirus em uma coorte de pacientes transplantados renais através da técnica de antigenemia [Mestrado]. Porto Alegre: UFRGS; 2001.

4. Osawa R, Singh N. Cytomegalovirus infection in critically ill patients: a systemic review. Crit Care. 2009;13(3)1-10.

5. Preiksaitis JK, Brennan CD, Fishman J, Allen U. Canidian Society of transplantation consensus workshop on cytomegalovirus management in solid organ transplantation final report. Am J Transplant. 2005; 5:218-27.

6. Schroeder RB. Antigenemia para citomegalovírus no pós-transplante renal: Escolha de um ponto de corte para o diagnóstico de doença citomegálica [Mestrado]. Porto Alegre: PUCRS; 2003.

7. Gunson RN, Collins T.C and. Carman W.F et al, Practical experience of high throughput real time PCR in the routine diagnostic virology setting. J Clin Virol $2006 ; 35,355-367$

8. Reina J, Ballesteros F, Gasco J, Munar M, Mari M. Usefulness of pp65 antigenemia and virema in the follow-up of renal transplant recipients with cytomegalovirus diseases treated with Ganciclovir. Diagnostic Microbiology and Infectious Disease. 2000;37,83-6

9. Van Der Bij W, Speich R: Management of citomegalovírus infection and disease after solid-organ transplantation. Clinical infectious disease. 2001;33(1):33-7.

10. Gerna, G., Revello, M.G., Percivalle, E., Morini, F. Comparison of different immunostaining techniques and monoclonal antibodies to the lower matrix phosphoprotein (pp65) for optimal quantitation of human cytomegalovirus antigenemia. J Clin Microbiology. 1992;30,1232-7.

11. Landry ML, Ferguson D., Cohen, S., Huber, K., and Wetherill, P. Effect of delayed specimen processing on Cytomegalovirus antigenemia test results. J Clin Microbiology. 1995;33,257-9

12. Ho SKN., Lo CY, Cheng IKP and ChanTM, Rapid cytomegalovirus pp65 antigenemia assay by direct Erythrocyte Lysis and Immunofluorescence Staining. J. Clin Microbiology. 1998;36,638-40

13. The Hauw, Arie P, Berg VD, Harmsen MC, Van Der Bij W. Van Son J. The cytomegalovirus antigenemia assay: Aplea for standardization. Scand J Infect Dis Suppl. 1995;99:25-29.

14. Grefte JM, Van der G, BTF., Schmolke, S., Van der GM, Van Son WJ et al. The lower matrix protein pp65 is the principal viral antigen present in peripheral blood leukocytes during an active cytomegalovirus infection. J Gen Virol. 1992;73,2923-32.
15. Revello MG, Percivalle, E., Di Matteo, A., Morini, F., Gerna, G. Nuclear expression of the lower matrix protein of human cytomegalovirus in peripheral blood leukocytes of immunocompromised viremic patients. J Gen Virol. 1992;73,437-42.

16. Schröeder R, mesko J, santos A, et al: cytomegalovirus antigenemia and renal function post-kidney-transplantation. Transplant Proc 31: 3027-8 1999.

17. Schroeder R, Michelon T, Fagundes I, et al: Cytomegalovirus disease latent and active infection rates during the first trimester after kidney transplantation. Transplantation Proceedings. 2004;36,896-8

18. Schroeder R, Michelon T, Fagundes I, et al: Antigenemia for Cytomegalovirus in Renal Transplantation: Choosing a Cutoff For the Diagnosis Criteria in Cytomegalovirus Disease. Transplantation Proceedings. 2005;37:2781-3.

19. Schröeder RB, Michelon T, Wurdig J, et al:. The Incidence of Cytomegalovirus Infection in Lung Transplant Recipients under Universal Prophylaxis with Intravenous Ganciclovir. The Brazilian Journal of Infectious Diseases. 2007;11(2):212-4.

20. Smith TF, Espy MJ, Mandrekar J et al: Quantitative real-time polymerase chain reaction for evaluating DNAemia due to Cytomegalovirus, Epstein-Barr virus, and BK virus in solid organs transplant recipients. Clin Infect Dis 2007;45:1056-61

21. Bressollette-Bodin C, Coste-Burel M, Besse B et al: Cellular normalization of viral DNA loads on whole blood improves the clinical management of cytomegalovirus or Epstein-Barr virus infections in the setting of pre-emptive therapy. J Med Virol 2009;81:90-8

22. Regina Schroeder Tatiana Michelon, Adamy R et al. Infecção por citomegalovírus no pós-transplante: comparação das técnicas de antigenemia pp65 por imunofluorescência e real time PCR. Anais do Congresso Brasileiro de Transplantes de órgãos. Recife 2009

23. Boeckh M, Boivin G: Quantitation of cytomegalovirus: methodologic aspects and clinical applications. Clinical Microbiology Reviews. 1998;11(3)533-54.

24. Kim DJ, Kim SJ, Park J, Choi GS, Lee S, et al. Real time PCR assay compared with antigenemia assay for detecting cytomegalovirus infection in cytomegalovírkidney transplant recipients. Transplantation Proceedings. 2007;39:1458-60.

25. Humar A, Lipton J, Welsh S, Moussa G, Messner H, Mazzulli T.A randomised trial comparing cytomegalovirus antigenemia assay vs screening bronchoscopy for the early detection and prevention of disease in allogeneic bone marrow and peripheral blood stem cell transplant recipients.Bone Marrow Transplant. 2001;28(5):485-90.

26. Schafer H, Bader P, Klingebiel T, Handgretinger R, Kanz L, Einsele H. Extramedullary relapses at uncommon sites after allogenic stem cell transplantation. Bone Marrow Transplant. 2000;26(10):1133-5

27. Dmitrienko S, YU A, Balshaw R, Shapiro RJ, Keown PA. The use of consensus guidelines for management of cytomegalovirus infection in renal transplantation. 2007;72,1014-22 